\title{
Prior context and the perception of lexically ambiguous sentences
}

\author{
V. M. HOLMES and R. ARWAS \\ University of Melbourne, Parkville, Victoria, Australia 9052 \\ and \\ M. F. GARRETT \\ Massachusetts Institute of Technology, Cambridge, Massachusetts 02139
}

\begin{abstract}
The perceptual complexity of lexically ambiguous and unambiguous sentences was compared in three experiments. In Experiment 1, the report of ambiguous words from rapidly presented ambiguous sentences was worse than the report of corresponding unambiguous words from unambiguous sentences. Results of Experiment 2 showed that the effect was not reduced by the presence of prior biasing context within the sentence. Experiment 3 repeated the finding with a sentence meaning classification task. It was concluded that both meanings of a lexically ambiguous sentence must be computed, even when prior context makes one meaning more plausible than the other.
\end{abstract}

An important factor in the process of comprehending a sentence is the determination of the appropriate meaning of the lexical items. It is usual for a lexical item not only to have multiple grammatical functions, but also to have more than one sense or meaning. Most lexical items have many senses, varying in the degree of semantic relatedness. Clearly, then, rapid selection has to be made between the possible senses of ambiguous words during sentence comprehension, the sense selected being the one that fits in best with the context in which the word occurs. It may be that contextual cues guide the choice of the most plausible sense without any alternative senses being considered, so that no extra processing time is required by the presence of the ambiguous item. On the other hand, it is possible that more than one sense must always be accessed and tested against the context, thereby resuiting in additional processing time. It was the intention of the present experiments to try to decide between these two hypotheses.

Evidence that the presence of a lexically ambiguous item in a sentence does increase perceptual complexity comes primarily from experiments using the phonememonitor task. Foss (1970), Foss and Jenkins (1973), and Cairns and Kamerman (1975) have shown that reaction times to detect a specified phoneme are longer immediately after ambiguous words than after corresponding unambiguous words. The difference is presumed

This research was partly supported by a grant from the Australian Research Grants Committee to the first author. The authors wish to thank Julia Vitale for her assistance in constructing the sentences for the first experiment and Katie Kirby for making up the anomalous items in the last experiment. Requests for reprints should be addressed to Dr. V. M. Holmes, Department of Psychology, University of Melbourne, Parkville, Victoria, Australia 3052. to reflect the increased computational load resulting from checking both senses against contextual information. Most other studies on lexical ambiguity have used tasks which do not reveal how the initial assignment of meaning to an ambiguous item occurs, since they require a variety of decisions to be made following comprehension of the sentence (Bever, Garrett, \& Hurtig, 1973; Cairns, 1973; Carey, Mehler, \& Bever, 1970; Foss, Bever, \& Silver, 1968; MacKay, 1966; Olson \& MacKay, 1974).

The study of the effects of context on the perception of lexically ambiguous sentences is similarly somewhat limited. Lackner and Garrett (1972) found that subjects' paraphrases of ambiguous sentences were influenced by relevant biasing context which was played softly in an unattended channel. This indicates that subjects must have been computing the alternative sentence meanings, in order for the biasing context to have had an effect. But it is not clear from this whether context controls the initial choice of the appropriate meaning, or operates to select one meaning after both have been accessed. Conrad's (1974) findings suggest that context has its influence after the alternative senses have been accessed. She found slower color naming responses for words presented after ambiguous sentences than for words presented after unrelated unambiguous sentences. The lexical interference effects occurred not only for the ambiguous word itself, but also for unambiguous control words that were made either appropriate or inappropriate by the preceding context. The results imply that more than one sense of an ambiguous word is activated during sentence comprehension, regardless of the previous context. Foss and Jenkins' (1973) finding that lexically ambiguous sentences, even with prior biasing context, were more complex perceptually than unambiguous control sentences, supports this interpretation. 
It seems that the available evidence goes against the view that context automatically determines the selection of the appropriate sense of an ambiguous lexical item without any consideration of the inappropriate sense. Nevertheless, the evidence indicating that biased ambiguous sentences take longer to process than unambiguous sentences, and that this is true regardless of context, is limited to one particular processing task, namely phoneme monitoring. The aim of the following experiments was, therefore: (1) to provide further tests of the perceptual effects of lexical ambiguities in unbiased sentences, and (2) to see whether any complexity effects are maintained in the presence of prior pragmatically biasing context.

\section{EXPERIMENT 1}

The basic question asked in this experiment was whether the presence of lexical ambiguity in both unbiased and contextually biased sentences would increase perceptual complexity. In all the ambiguous sentences used, at least two interpretations were semantically possible. The division into unbiased and biased sentences was based on a paraphrasing pretest which provided the relative probabilities with which a given interpretation was consciously reported.

The task chosen to provide an index of complexity in sentence perception was rapid serial visual presentation (RSVP) (Forster, 1970). In this task, subjects are presented with individual words appearing successively on the same part of a screen at very fast rates. If presentation rate is fast enough, it outpaces the subject's ability to identify all the words, and the subject is only able to report some of the words. Forster (1970) and Forster and Ryder (1971) have shown that the number of words reported is a function of the degree to which the subject can organize the input syntactically and semantically.

The ambiguous and unambiguous sentences used in the experiment differed only by the presence of one word. That is, sentences were constructed in triads, for example, "They agreed that the (seal/cat/glue) would be useful." Hence, two measures of perceptual complexity could be derived: (1) the percentage of ambiguous or control (target) words that were reported, and (2) the percentage of remaining common (context) words that were reported. In this way, it was hoped to determine more precisely the locus of any complexity effect.

\section{Method}

Design and materials. Seventy-five lexically ambiguous sentences, each eight words in length, were constructed so that two alternative interpretations seemed reasonably plausible and likely. The sentences were randomly ordered and were interspersed with 15 superficially similar unambiguous sentences. The list was then presented to pretest subjects, whose paraphrases yielded the data for the relative probabilities of the interpretations. Twenty-four of the sentences were quite
Table 1

Examples of Sentences Used in Experiment 1*

Unbiased Sentences:

The powerful punch/brandy/kick made the man feel weak.

They didn't want the iron/radio/metal they had ordered.

We all though that the batter/pastry/umpire was mediocre.

The visitors complained that the port/sherry/harbor was awful.

\section{Biased Sentences:}

The child saw the bat/mouse/glove in the basement.

The customer noticed that the scales/shelves/fins looked peculiar.

They agreed that the priest's habits/beliefs/shoes were conservative.

Helen was bothered by the cold/heat/pain all day.

${ }^{*}$ For the target words, which are in italics, the ambiguous word is listed first, then the more likely alternative, then the less likely alternative.

unbiased or neutral, that is, one alternative was expressed $50 \%-60 \%$ of the time and the other $40 \%-50 \%$ of the time. The average preference for one alternative in these sentences was $54 \%$. From the remaining sentences, 26 of the "most natural" sentences were chosen, such that the average bias was $78 \%$.

For each of the 50 ambiguous sentences, two unambiguous control sentences were constructed, one corresponding to the more likely alternative and the other corresponding to the less likely alternative. Examples of some of the sentences used are shown in Table 1, which shows that the control sentences were exactly the same as their ambiguous counterparts except for the presence of the unambiguous word. The target words were equated for length in letters (within two letters). The average lengths in letters for target words in the 24 unbiased sentences were: ambiguous, 5.3; more likely control, 5.5; less likely control, 5.5. In the 26 biased sentences, average word lengths were: ambiguous, 5.4; more likely control, 5.6; less likely control, 5.3. The frequency of occurrence of the words was roughly equated across triads, according to Kučera and Francis (1967). The average frequencies per million for unbiased sentences were: ambiguous, 70; more likely control, 73; less likely control, 75 . The average frequencies per million for biased sentences were: ambiguous, 74; more likely control, 70; less likely control, 81 . The subjects design was therefore a threefactor design, with groups, bias, and ambiguity as the factors. In this experiment, as well as in Experiments 2 and 3 , statistical decisions were based on a Type I error rate of $\alpha=.01$. In the analyses of all three experiments, the groups factor and its interaction with other factors were at no time significant.

Procedure. The 50 sentence triads were randomized and three lists were compiled so that one of the matched sentences occurred in each list. For each list, one-third of the sentences were ambiguous and two-thirds were unambiguous. Twelve sequences with missing words were distributed throughout each list, and several practice items were included. The sentences from the lists were filmed on 16-mm movie film, with one word per frame. Sentences were preceded by a blank interval followed by the word "READY." The film was projected at a rate of 16 words/sec. At the end of each sequence, subjects were instructed to write down as many words as possible in the correct order without guessing. This procedure is essentially the same as that used by Forster (1970), Forster and Ryder (1971), and Holmes and Watson (1976). The session concluded with subjects being given a typed list of all the test sentences with which they had been presented interspersed with 12 padding items. The subjects rated the sentences for the plausibility of the events described, using a 5-point scale, where +2 represented "perfectly ordinary and normal events," and -2 represented "very bizarre and unlikely events." 
Subjects. Subjects for the pretest were 20 students from Wellesley College and 20 M.I.T. students, who were paid for their participation. Forty eight different M.I.T. students were paid to act as subjects in the RSVP experiment, in which they were tested in groups of one to four.

\section{Results and Discussion}

The percentage of target words reported in each condition was calculated for both subjects and sentences. In addition, the average numbers of words reported from the remaining seven context words were determined and converted to percentages. The means for the two measures are shown in Table 2. It can be seen that the percentage of context words reported is much higher than that of target words. This can be explained by the serial position effect obtained with the task (Forster, 1970). The target words generally occurred in the middle serial positions, which are less accurately reported than the initial and final serial positions of the context words.

Analyses of variance were performed separately for target and context word scores. Instead of the overall main effect of ambiguity, two orthogonal planned contrasts were tested. For the target words, ambiguous words were significantly more poorly reported than were unambiguous words in the subjects analysis $[\mathrm{F}(1,90)=18.67, \quad \mathrm{MSe}=179.69]$, although in the sentences analysis, the effect failed to achieve significance $[F(1,98)=3.85, \quad \mathrm{MSe}=439.91]$. Note that there was no significant difference in the report of unambiguous words for more likely and less likely interpretations $(F<1$ in both subjects and sentences analyses). Target words were better reported in biased than in unbiased sentences; this was significant by subjects $[\mathrm{F}(1,45)=15.98, \quad \mathrm{MSe}=174.80]$, though not by sentences $(F<1)$. In neither analysis was there an interaction between bias and ambiguity $(F<1$ in both cases). For the context words, there was no significant difference between the ambiguous and unambiguous sentences, nor within the two unambiguous interpretations. Context words were better reported in biased than in unbiased sentences; this was significant by subjects $[F(1,45)=8.09, \mathrm{MSe}=22.22]$,

Table 2

Mean Percentage of Target and Context Words Reported in Experiment 1

\begin{tabular}{lccc}
\multicolumn{1}{c}{ Bias } & Ambiguous & $\begin{array}{c}\text { More Likely } \\
\text { Alternative }\end{array}$ & $\begin{array}{c}\text { Less Likely } \\
\text { Alternative }\end{array}$ \\
\cline { 2 - 4 } & \multicolumn{3}{c}{$\begin{array}{c}\text { Target Words } \\
\text { Unbiased }\end{array}$} \\
Biased & 28.1 & 34.0 & 33.1 \\
Total & 32.0 & 40.6 & 41.3 \\
& 30.0 & 37.3 & 37.2 \\
Unbiased & 52.0 & Context Words \\
Biased & 53.2 & 50.1 & 51.7 \\
Total & 52.6 & 50.7 & 53.8 \\
\hline
\end{tabular}

Table 3 Mean Plausibility Ratings for Sentences Used in Experiment 1

\begin{tabular}{lcccc}
\hline & \multicolumn{4}{c}{ Ambiguity } \\
\cline { 2 - 4 } \multicolumn{1}{c}{ Bias } & Ambiguous & $\begin{array}{c}\text { More Likely } \\
\text { Alternative }\end{array}$ & $\begin{array}{c}\text { Less Likely } \\
\text { Alternative }\end{array}$ & Total \\
\hline Unbiased & .57 & .63 & .78 & .66 \\
Biased & .66 & .65 & .49 & .60 \\
Total & .62 & .64 & .64 & \\
\hline
\end{tabular}

but not by sentences $(F<1)$. The interaction between bias and ambiguity was again not significant in either analysis (both $\mathrm{Fs}<1$ ).

Plausibility ratings for the sentences indicated that all conditions contained sentences of comparable plausibility. The means of the plausibility ratings are shown in Table 3. Ratings of this magnitude represent judgments that the sentences depict situations somewhere between "just normal" and "quite normal and ordinary." The correlation between mean plausibility rating and targetword score over the 150 sentences was +.197 , which, although statistically significant, is rather small. The small magnitude of the correlation presumably arises from the lack of marked variation in the plausibility scores. Therefore, it may be concluded that any differences observed in perceptual complexity between sentence types are not attributable to differences in the plausibility of the sentences.

On the basis of the results of the subjects analyses, it seems that ambiguous sentences do provide additional processing complexity, in that the ambiguous words themselves are not reported as accurately as their unambiguous control words. The absence of any interaction between this effect and the bias of the sentences demonstrates that the result holds true for sentences which have a strong bias to one interpretation as well as for sentences which are relatively unbiased. However, the fact that the ambiguity effect did not reach significance in the sentences analysis indicates that not all sentences were contributing to the result, and that the effect may not be a general feature of all lexically ambiguous sentences. The subjects analyses also indicated that both target and context words were better reported in biased than in unbiased sentences. The total lack of the bias effect in the sentences analyses suggests that even fewer sentences were responsible for this result.

\section{EXPERIMENT 2}

The second experiment was designed to determine whether the ambiguity effect observed in Experiment 1 was specific to the sentences used in that experiment, or whether it could be obtained with a different set of sentences. The role of biasing context was also examined again. For the biased sentences in Experiment 1, it was not possible in many cases to decide whether the preceding or the subsequent context had led subjects to 
favor one concious interpretation. In Experiment 2, sentences were investigated in which the "disambiguation" clearly came from the prior context.

Presumably neutral sentences were constructed (e.g., "The tourist exclaimed that the record was remarkable,") and were biased to one interpretation by changing the initial noun (e.g., "tourist" was replaced with "singer"). The two resulting sentences were compared with unambiguous control sentences (e.g., in which "music" was substituted for "record"). Thus, the main interests were again an overall ambiguity effect and a possible interaction of the effect with the nature of the context. Comprehension difficulty was again assessed by means of the RSVP task.

\section{Method}

Design and materials. Forty sets of sentences were constructed so that the following four conditions were exemplified: ambiguous-biased, ambiguous-un biased, unambiguous-biased, unambiguous-unbiased. Examples of some of the sentence sets are given in Table 4. Nine of the sentences were seven words long and the remainder were eight words long. The target words within a given sentence set varied in length by a maximum of two letters. The mean length in letters was 5.0 for ambiguous words and 5.2 for unambiguous words. The initial nouns were also equated for length, the means being 7.1 letters for biasing words and 7.2 for neutral words. The average frequencies, according to Kučera and Francis (1967), were 76 for ambiguous words and 88 for unambiguous words. It was not possible to equate for frequency of the initial nouns, since the neutral words were usually more general words, and therefore more frequently used. The average frequency was 36 for biasing words and 130 for neutral words. For 34 of the 40 sentence sets, an ambiguous word from Experiment 1 was used, but with different sentence contexts and different unambiguous controls. In order to obtain an estimate of the validity of the assignments of sentences to biased or unbiased categories, subjects were asked to paraphrase the ambiguous sentences.

Procedure. Four lists of sentences containing one version of each sentence set were prepared, and each list was given to a separate group of subjects. Two sentences from each of the four conditions occurred randomly within each of five blocks of sentences. Two padding sequences with words missing were also included in each block. Thus, the subject was presented with 50 items, preceded by nine practice items. The stimuli were

\section{Table 4}

Examples of Sentences Used in Experiment 2*

B: The team said that two matches/contests were enough. $\mathrm{U}$ : The boys said that two matches/contests were enough.

B: The managers met near the bank/shop last night.

$\mathrm{U}$ : The athletes met near the bank/shop last night.

B: The customer accepted the change/money with pleasure.

$\mathrm{U}$ : The writer accepted the change/money with pleasure.

B: The teacher affected my pupils/students very badly.

$U:$ The light affected my pupils/students very badly.

B: The hostess expected the company/friends to leave.

U: The neighbor expected the company/friends to leave.

B: The musicians agreed that John's pitch/voice was perfect. U: The spectators agreed that John's pitch/voice was perfect.

${ }^{*} B$ indicates the biased and $U$ the unbiased version. The ambiguous word is listed first followed by the unambiguous control.
Table 5

Mean Percentage of Target Words Reported in Experiment 2

\begin{tabular}{lcc} 
& \multicolumn{2}{c}{ Ambiguity } \\
\cline { 2 - 3 } Context & Ambiguous & Unambiguous \\
\hline Biased & 38.7 & 43.1 \\
Unbiased & 32.5 & 39.0 \\
Total & 35.6 & 41.1 \\
\hline
\end{tabular}

presented by means of a PDP-11 computer on an oscilloscope screen. The words were presented one at a time, as in Experiment 1, but this time each word was preceded and followed by a masking stimulus of a matrix of dots. Holmes and Forster (1972) have shown that this procedure virtually eliminates the word-length effect found with RSVP (Forster, 1970). A subject initiated each trial by pressing a button. A warning signal appeared briefly, followed $.5 \mathrm{sec}$ later by the mask for $40 \mathrm{msec}$, then the first word for $40 \mathrm{msec}$, and so on. The overall rate was therefore just over 14 words/sec. Subjects were instructed to write down as many words as possible, in the correct order, without guessing.

For the bias ratings, two lists of the sentences were compiled, each containing either the biased or unbiased version of an ambiguous sentence. A given list contained half of the biased and half of the unbiased versions. The sentences were randomized, and 13 similar unambiguous sentences were interspersed throughout each list. Subjects were instructed to rewrite the sentences in their own words and appropriate examples were given.

Subjects. For the RSVP task, subjects were 52 undergraduates at the University of Melbourne. A separate group of 40 undergraduates served as subjects for the paraphrasing task. All subjects were paid for their participation.

\section{Results and Discussion}

The means of the percentages of target words reported are given in Table 5, which shows that ambiguous words were more poorly reported than unambiguous words. This was significant by subjects $[F(1,48)=9.77, \quad M S e=159.94]$, though not by sentences $[F(1,39)=1.73$, MSe $=694.80]$. Target words in biased sentences were better reported than those in unbiased sentences. This was significant by subjects $[\mathrm{F}(1,48)=7.35, \mathrm{MSe}=183.65]$ and approached significance by sentences $[F(1,39)=4.45, M S e=233.19]$. The interaction between the two factors was not significant ( $F<1$ in both subjects and sentences analyses). The mean percentages of context words reported are shown in Table 6. For the initial biasing word, which was the same for ambiguous and unambiguous sentences, but different for the biased and unbiased versions, there were no significant effects in the subjects analysis, or in the sentences analysis (all Fs $<1$ ). Similarly, there were no significant components in the analyses of the context words common to all four versions of a sentence.

The check on the bias of the sentences revealed that for biased sentences, an average of $94 \%$ of subjects' paraphrases expressed the predicted interpretation. In contrast, the average bias of the unbiased sentences was $72 \%$. However, the latter figure is not as low as might be expected; it seemed to be more difficult in this experiment to design sentences for which subjects were about 
Table 6

Mean Percentage of Context Words Reported in Experiment 2

\begin{tabular}{|c|c|c|}
\hline \multirow[b]{2}{*}{ Context } & \multicolumn{2}{|c|}{ Ambiguity } \\
\hline & Ambiguous & Unambiguous \\
\hline & \multicolumn{2}{|c|}{ Initial Biasing Word } \\
\hline $\begin{array}{l}\text { Biased } \\
\text { Unbiased }\end{array}$ & $\begin{array}{l}63.8 \\
67.9\end{array}$ & $\begin{array}{l}65.4 \\
68.1\end{array}$ \\
\hline \multirow[t]{2}{*}{ Total } & 65.9 & 66.7 \\
\hline & \multicolumn{2}{|c|}{ Common Context Words } \\
\hline $\begin{array}{l}\text { Biased } \\
\text { Unbiased }\end{array}$ & $\begin{array}{l}53.7 \\
53.6\end{array}$ & $\begin{array}{r}55.4 \\
55.9\end{array}$ \\
\hline Total & 53.7 & 55.6 \\
\hline
\end{tabular}

equally as likely to report either interpretation in their paraphrases. It is noteworthy that Foss and Jenkins (1973) had even less success in creating neutral ambiguous sentences: The average bias rating of their unbiased sentences was $78 \%$.

As was found for Experiment 1, the target-word ambiguity effect obtained in this experiment was significant by subjects but not by sentences. In an attempt to overcome this problem, an items analysis was performed in which the target-word data from both experiments were pooled. For each Experiment 1 item, there was a score of percentage of subjects reporting the word for the ambiguous version, and an average over the two unambiguous versions. For each Experiment 2 item, the average over the two context versions was calculated for ambiguous and unambiguous sentences. A related-measures $t$ test showed that the mean of the ambiguous items (32.7) was significantly different from that of the unambiguous items $(39.2)[t(89)=2.40]$. Hence, it was concluded that, with a large enough sample of sentences, the ambiguity effect can be shown to be generalizable.

The results also indicated that prior biasing context does not reduce the difficulty in processing produced by the presence of lexical ambiguity. Furthermore, when the sentences contained an initial noun that was biasing, as opposed to neutral, target words tended to be better reported whether they were unambiguous or ambiguous.

\section{EXPERIMENT 3}

The aim of Experiment 3 was simply to see if yet another sentence perception task, namely sentence meaning classification, would produce an ambiguity effect. In the task, the time taken to decide that a sentence is meaningful has been assumed to indicate the subject's difficulty in determining the structure and meaning of the sentence (Dooling, 1972; Forster \& Olbrei, 1973; Mistler-Lachman, 1972). This task was used in the present experiment, with exactly the same stimulus sentences as were used in Experiment 2. Thus, the factors of ambiguity and prior biasing context and the possible interaction of the two were investigated again with a different paradigm.
Method

Design and procedure. Since the test sentences were the same as those used in Experiment 2, the design was the same. Semantically anomalous distractor items, which were of similar surface structure to the test sentences, were constructed for inclusion in each of the four lists. There were 30 distractors, 6 within each block of eight test items. Examples of some of the distractors are: The graziers locked their diseases in the cupboard. The cook showed that the oil was broken. Several artists sold the paintings to the bricks. The lecturers discovered that the hypothesis was angry.

The stimuli were presented on an oscilloscope screen controlled by the PDP-11 computer. The words of a sequence appeared simultaneously across the screen when the subject pressed a "go" button. Subjects were instructed to read the sequence of words carefully and to decide quickly whether it formed a completely meaningful sentence. They were to press the "yes" button if the sequence was a meaningful sentence and the "no" button if it was not. Stimulus presentation was terminated either by the depression of one of the buttons or after $8 \mathrm{sec}$. Response times were measured from the onset of the stimulus sequence to the subject's button press. Several examples of nonmeaningful sequences were given in the instructions, so that subjects were well aware of the decision they were to make. There were 13 practice items.

At the completion of the sentence classification task, subjects were required to judge the plausibility of the test items. Instead of rating plausibility on a scale, subjects were asked to compare pairs of sentences containing the ambiguous and unambiguous versions and to indicate which of the two sentences was more plausible, or whether the two seemed equally plausible. In each list, 12 dummy pairs of items were included in which one of the pair was clearly much more bizarre than the other.

Subjects. Subjects were 52 paid volunteers who were students at the University of Melbourne. Data from two subjects who misclassified more than $20 \%$ of the sentences as nonsentences were discarded.

\section{Results and Discussion}

An average of $4.7 \%$ of the sentences were incorrectly classified by the subjects. Reaction times for the errors were ignored. In a further $.2 \%$ of cases, the subject failed to respond within $8 \mathrm{sec}$. In order to diminish the influence of any particularly long reaction times, the standard deviation of a given subject's correct "yes" responses was computed, and any score which exceeded two standard deviations from the mean was set at that value. This procedure affected $3.5 \%$ of the scores. The adjusted scores were averaged over sentences for each subject and over subjects for each sentence. The means for each condition (in milliseconds) are shown in Table 7. Ambiguous sentences were classified more slowly than were unambiguous sentences; this was significant by subjects $[\mathrm{F}(1,51)=12.52, \mathrm{MSe}=38,681.9]$, but not by sentences $[F(1,39)=2.27, \mathrm{MSe}=115,062.2]$.

Table 7

Mean Classification Times (in Milliseconds) in Experiment 3

\begin{tabular}{lcc} 
& \multicolumn{2}{c}{ Ambiguity } \\
\cline { 2 - 3 } Context & Ambiguous & Unambiguous \\
\hline Biased & 2747 & 2673 \\
Unbiased & 2840 & 2720 \\
Total & 2793 & 2697 \\
\hline
\end{tabular}


There was a trend for biased sentences to be classified more quickly than unbiased sentences, but this was significant neither by subjects $[\mathrm{F}(1,51)=5.45$, $\mathrm{MSe}=46,840.6]$ nor by sentences $[\mathrm{F}(1,39)=2.36$, $\mathrm{MSe}=62,217.3]$. The interaction of ambiguity and context was not significant in either analysis (both Fs $<1$ ).

According to the subjects analysis, ambiguous sentences were more difficult to process than the unambiguous controls, and the difference was not reduced by prior biasing context within the sentence. This result, based on a new sample of subjects and a different perceptual processing task, agrees entirely with the target-word effect found in the last experiment. Nevertheless, the nonsignificance of the sentences analysis again implies that only some sentences were causing the subjects difference. If a small group of atypical sentences produced the subjects ambiguity effect, it might be expected that the same unusual items were the cause of the effect in both experiments. Thus, items were classified with respect to whether they produced an effect in one experiment and whether they produced an effect in the other. The relationship was not significant for biased or unbiased sentences $\left[\chi^{2}(1)=1.07\right.$ and 1.82 , respectively $]$. The result is also borne out by correlations of the magnitudes of the differences between sentence pairs for target-word scores and classification times. For biased sentences, the correlation was -.082 , and for unbiased sentences, the correlation was -.052 . Needless to say, with 38 degrees of freedom, the correlations were not significant. It is apparent that it cannot be the same unusual sentences that created the ambiguity effect in the two experiments.

The results of the plausibility comparisons are shown in Table 8 . On the average, over $50 \%$ of the subjects judged the ambiguous and unambiguous versions to be equally plausible. Of the remaining judgments, ambiguous and unambiguous versions were preferred to about the same extent; if anything, the ambiguous sentences were considered to be more plausible. This indicates that the superior performance on unambiguous sentences in both Experiments 2 and 3 is not due to greater plausibility in meaning.

Table 8

Mean Percentage of Subjects Judging Ambiguous or Unambiguous Version of Sentence Pairs as

More Plausible, for Sentences Used in Experiments 2 and 3

\begin{tabular}{lccc}
\hline Context & $\begin{array}{c}\text { Ambiguous } \\
\text { Version } \\
\text { Preferred }\end{array}$ & $\begin{array}{c}\text { Unambiguous } \\
\text { Version } \\
\text { Preferred }\end{array}$ & $\begin{array}{c}\text { No } \\
\text { Preference }\end{array}$ \\
\hline Biased & 21.7 & 16.9 & 61.4 \\
Unbiased & 22.7 & 19.1 & 58.2 \\
Mean & 22.2 & 18.0 & 59.8 \\
\hline
\end{tabular}

\section{GENERAL DISCUSSION}

Taken together, the results of the present experiments show that perceptual processing of ambiguous and unambiguous sentences is not equivalent; the presence of a lexically ambiguous item does complicate the perception of a sentence. Moreover, prior biasing context within the sentence does not reduce the complexity caused by the ambiguous lexical item. The results, obtained with different experimental tasks, are consistent with the phoneme-monitor results of Foss (1970), Foss and Jenkins (1973), and Cairns and Kamerman (1975). Any attempt to explain the observed effects involves the postulation of a mental lexicon whose entries are scanned during sentence comprehension. The work of Jastrzembski and Stanners (1975), Rubenstein, Garfield, and Millikan (1970), and Rubenstein, Lewis, and Rubenstein (1971) suggests that unambiguous items are represented by one lexical entry, whereas ambiguous items are represented by separate entries for each sense. Thus, the person trying to comprehend a two-way ambiguous sentence would need to locate both entries for the ambiguous item and check the contents of each, before selecting the sense that seemed more appropriate for the context.

The exact nature of the processing operations would likely be dependent upon particular task requirements. In the first two experiments reported here, in which the RSVP task was used, subjects were forced to identify each word as it arrived, because of the extremely rapid presentation rate, and because of the masking that prevented lagging behind the input. It is important to note that it was performance on the ambiguous target words that suffered as a result of the procedure, and not performance on the context words. It seems that on encountering an ambiguous item, subjects may have located one entry, noted that another entry needed to be checked as well, and attempted to do so. However, under pressure of identifying subsequent words, they may not have had time to code either of the meanings adequately, which would account for the fact that the subject was then unable to report the ambiguous item at all. Subjects could adopt a strategy of identifying the ambiguous words at the expense of the context words, but it appears that this is not what they did.

The complexity effect observed with the meaning classification task in Experiment 3 presumably involves steps subsequent to the accessing of two lexical entries for the ambiguous item. Here, subjects have as long as they need to determine the meaning of the sentence; they are not required to identify each item in a constant very brief period. Having located both entries of an ambiguous item, subjects would probably examine the contents of both entries and try to integrate them with the surrounding context. At which stage subjects would select one of the interpretations over the other is not 
clear. In the present experiment, the two interpretations were semantically possible, even in the biasing context condition, so neither interpretation actually needed to be rejected for a correct response to be made.

The present experiments have also shown that the additional processing steps required for ambiguous sentences were not short-circuited by the presence of prior pragmatically biasing context. Such a contextindependent lexical "look-up" procedure is also implied by Conrad's (1974) results. However, at the same time, sentences with biasing initial nouns tended to be less complex than sentences with neutral initial nouns, whether the subsequent target word was ambiguous or not. This suggests that a biasing word guides the subject to the semantically associated target word more rapidly than does a neutral low-associated word. The effect might be expected on the basis of Meyer and Schvaneveldt's (1971) finding that lexical decisions are faster for pairs of associated words than for pairs of unassociated words. What is interesting here is that, even though the associated target-word entry is found more quickly, if it is ambiguous, the subject still tries subsequently to locate the alternative less associated entry. Subjects appear to be acting on the premise that a relatively implausible sentence meaning may be intended and that this meaning ought therefore to be computed as well.

The major unresolved finding in the present results is the fact that the ambiguity effect in individual experiments was significant only in the subjects analyses and not in the items analyses. Clark (1973) has argued against accepting conclusions based on subjects analyses alone without determining how large the effect is relative to the variance within items. The effects observed in Experiments 1 and 2 were only strong enough to be significant when considered in relation to the variance of all 90 items. Normally, the requirement that such a large number of items be used would be unnecessarily onerous. However, it seems unlikely that a small number of atypical items were responsible for the effect in each of the experiments, in view of the lack of correlation between items producing an effect in Experiment 2 and those producing an effect in Experiment 3. If there had been a constant set of items causing both effects, some classification of the items might have been possible. For example, Hogaboam and Perfetti (1975) have argued that lexical ambiguity effects might only occur when the ambiguous words differ widely in the frequency with which each sense occurs, and when the sentence context biases the interpretation to the less frequent sense. The possibility that there is an indentifiable characteristic of items that produce a strong ambiguity effect needs to be pursued, but there is no positive evidence for this in the present results. On the basis of the experiments reported here, it is concluded that the lexical ambiguity effect is certainly real, but that it is not a particularly large effect in comparison to other sources of variation in sentence processing.

\section{REFERENCES}

Bever, T., Garrett, M. F., \& Hurtig, R. The interaction of perceptual processes and ambiguous sentences. Memory \& Cognition, 1973, 1, 277-286.

CalRNs, H. S. Effects of bias on processing and reprocessing of lexically ambiguous sentences. Journal of Experimental Psychology, 1973, 97, 337-343.

CaIrNs, H. S., \& Kamerman, J. Lexical information processing during sentence comprehension. Journal of Verbal Learning and Verbal Behavior, 1975, 14, 170-179.

Carey, P. W., Mehler, J., \& Bever, T. G. Judging the veracity of ambiguous sentences. Joumal of Verbal Learning and Verbal Behavior, 1970, 9, 243-254.

Clark, H. H. The language-as-fixed-effect fallacy: A critique of language statistics in psychological research. Journal of Verbal Learning and Verbal Behavior, 1973, 12, 335-359.

Conrad, C. Context effects in sentence comprehension: A study of the subjective lexicon. Memory \& Cognition, 1974, 2, $130-138$.

Dooling, D. J. Some context effects in the speeded comprehension of sentences. Journal of Experimental Psychology, 1972, 93, 56-62.

Forster, K. I. Visual perception of rapidly presented word sequences of varying complexity. Perception \& Psychophysics, $1970,8,215-221$.

Forster, K. I., \& OlbreI, I. Semantic heuristics and syntactic analysis. Cognition. 1973, 2, 319-347.

Forster, K. I., \& RYDer, L. A. Perceiving the structure and meaning of sentences. Journal of Verbal Learning and Verbal Behavior, 1971, 10, 285-296.

Foss, D. J. Some effects of ambiguity upon sentence comprehension. Journal of Verbal Learning and Verbal Behavior, 1970, 9, 699-706.

Foss, D. J., Bever, T. G., \& Silver, M. The comprehension and verification of ambiguous sentences. Perception \& Psychophysics, 1968, 4, 304-306.

Foss, D. J., \& Jenkans, C. M. Some effects of context on the comprehension of ambiguous sentences. Journal of Verbal Learning and Verbal Behavior, 1973, 12, 577-589.

hogaboam, T. W., \& Perfetti, C. A. Lexical ambiguity and sentence comprehension. Journal of Verbal Learning and Verbal Behavior, 1975, 14, 265-274.

Holmes, V. M., \& Forster, K. I. Perceptual complexity and underlying sentence structure. Journal of Verbal Learning and Verbal Behavior, 1972, 11, 148-156.

Holmes, V. M., \& Watson, I. J. The role of surface order and surface deletion in sentence perception. Quarterly Journal of Experimental Psychology, 1976, 48, 155-165.

Jastrzembski, J. E., \& Stanners, R. F. Multiple word meanings and lexical search speed. Journal of Verbal Leaming and Verbal Behavior, 1975, 14, 534-537.

Kúčera, H., \& Francis, W. Computational analysis of present-day American English. Providence: Brown University Press, 1967.

LACKNER, J. R., \& GARRETT, M. F. Resolving ambiguity: Effects of biasing context in the unattended ear. Cognition, 1972, 1, 359-372.

Mackay, D. G. To end ambiguous sentences. Perception \& Psychophysics, 1966, 1, 426-436.

MeYer, D. E., \& Schvaneveldt, R. W. Facilitation in recognizing pairs of words: Evidence of a dependence between retrieval operations. Journal of Experimental Psychology, 1971, 90, 227-234. 
Mistler-Lachman, J. L. Levels of comprehension in processing of normal and ambiguous sentences. Journal of Verbal Learning and Verbal Behavior, 1972, 11, 614-623.

Olson, J. N., \& MacKaY, D. G. Completion and verification of ambiguous sentences. Journal of Verbal Learning and Verbal Behavior, 1974, 13, 457-470.

Rubenstein, H., Garfield, L., \& Millikan, J. A. Homographic entries in the internal lexicon. Journal of Verbal Learning and Verbal Behavior, 1970, 9, 487-494.
Rubenstein, H., Lewis, S. S, \& Rubenstein, M. A. Homographic entries in the internal lexicon: Effects of systematicity and relative frequency of meanings. Journal of Verbal Learning and Verbal Behavior, 1971, 10, 57-62.

(Received for publication February 17, 1976; revision received July 13,1976 .) 